\title{
Temperatura base inferior, soma térmica e fenologia de cultivares de videira e quivizeiro
}

\author{
Lower base temperature, thermal time and grapevine phenology and kiwi cultivars
}

\author{
Rafael Anzanello ${ }^{\text {* }}$ \& Mariane Castanho de Christo \\ ${ }^{1}$ Secretaria da Agricultura, Pecuária e Desenvolvimento Rural, Veranópolis, RS, Brasil. *Autor para correspondência:rafael- \\ anzanello@seapdr.rs.gov.br \\ ${ }^{2}$ Universidade de Caxias do Sul, Caxias do Sul, RS, Brasil.
}

Submissão: 27/06/2018 / Aceite: 05/04/2019

\begin{abstract}
RESUMO
Este estudo objetivou avaliar a temperatura-base inferior (Tb), soma térmica e fenologia de cultivares de videira e de quivizeiro. Estacas com $25-35 \mathrm{~cm}$ das cultivares de videira Chardonnay (CH), Isabel (IS), Niágara Branca (NB), Concord (CO) e Bordô (BO) e de quivizeiro Bruno (BR), Monty (MO), Elmwood (EL), MG06 (MG) e Yellow Queen (YQ) foram coletadas em pomares localizados em Veranópolis, RS, em 03/06/2015. As estacas intactas foram submetidas a $0{ }^{\circ} \mathrm{C}$ por 1.008 Horas de Frio (HF), em câmaras incubadoras para a superação da dormência, embaladas em filme plástico preto. Na sequência, foram transferidas para as temperaturas de $4,6,8,10$ e $12{ }^{\circ} \mathrm{C}$, em estacas de nós-isolados, plantadas em espuma fenólica. Durante 150 dias, as gemas foram avaliadas a cada 2-3 dias quanto à brotação, no estádio de ponta verde e seus dados (1/dias para brotação) inseridos em curvas de regressão para estimativa da Tb para cada genótipo. Séries históricas de fenologia de 10 anos das cultivares e dados meteorológicos dos locais de cultivo foram utilizados para o cálculo da soma térmica (graus-dia) das frutíferas durante o ciclo vegetativo. A Tb se diferenciou entre as espécies frutíferas. A Tb foi menor para cultivares de quivizeiro $\left(B R=3,0{ }^{\circ} \mathrm{C} ; \mathrm{MO}=3,3{ }^{\circ} \mathrm{C}\right.$; $\mathrm{EL}=3,1{ }^{\circ} \mathrm{C} ; \mathrm{MG}=3,2{ }^{\circ} \mathrm{C}$ e $\mathrm{YQ}=3,0{ }^{\circ} \mathrm{C}$ ) e maior para cultivares de videira $\left(\mathrm{CH}=4,2{ }^{\circ} \mathrm{C}\right.$; IS $=4,3^{\circ} \mathrm{C} ; \mathrm{NB}=4,1^{\circ} \mathrm{C} ; \mathrm{CO}=6,2^{\circ} \mathrm{C}$ e $\left.\mathrm{BO}=4,4^{\circ} \mathrm{C}\right)$. A soma de graus-dia (GD) variou de 1670,9 a 2060,7 para as cultivares de videira e de 3179,6 a 3762,0 para as cultivares de quivizeiro. A maior soma de GD para a cultura do quivizeiro é dada pelo maior número de dias do seu ciclo vegetativo associada a menor $\mathrm{Tb}$ dos genótipos, se comparada à cultura da videira. O subperíodo fenológico (brotação à maturação) das frutíferas, em 100\% dos casos, respondeu mais ao tempo térmico (graus-dia) do que ao tempo cronológico (dias) para completar o ciclo vegetativo.
\end{abstract}

PALAVRAS-CHAVE: ciclo vegetativo, dormência, graus-dia, Actinidia sp., Vitis sp.

\begin{abstract}
This study aimed to evaluate the lower base temperature (Tb), thermal time and grapevine phenology and kiwi cultivars. Twigs 25-35-cm long for the following cultivars: grapevine, Chardonnay (CH), Isabel (IS), Niágara Branca (NB), Concord (CO) and Bordô (BO); and kiwi, Bruno (BR), Monty (MO), Elmwood (EL), MG06 (MG) and Yellow Queen (YQ) were collected in orchards in Veranópolis, RS State, on 06/03/2015. Intact twigs packed in black plastic film were subjected to 1,008 chilling hours $(\mathrm{HC})$ at $0^{\circ} \mathrm{C}$ in incubators to overcome dormancy and then transferred to temperatures of $4,6,8,10$, and $12^{\circ} \mathrm{C}$ on single-node cuttings planted in phenolic foam. Over $150 \mathrm{~d}$, budburst of the buds was evaluated in 2-3-d intervals in the greentip stage. The resulted inverse data for number of days to budburst (1/days to budburst) was inserted into regression curves to estimate Tb for each genotype. Historical phonological series comprised of 10-years for the analyzed cultivars and meteorological data of the cultivation sites were used to determine thermal time (degree-days) for the fruit trees during the growing season. Temperate fruit species exhibited different Tb. Tb was lower for kiwi cultivars ( $B R=3,0^{\circ} \mathrm{C} ; \mathrm{MO}=3,3^{\circ} \mathrm{C} ; \mathrm{EL}=3,1^{\circ} \mathrm{C} ; \mathrm{MG}=3,2^{\circ} \mathrm{C}$ and $\mathrm{YQ}=3^{\circ} \mathrm{C}$ ) and higher for grapevine cultivars $\left(\mathrm{CH}=4,2^{\circ} \mathrm{C}\right.$; IS $=4,3^{\circ} \mathrm{C} ; \mathrm{NB}=4,1^{\circ} \mathrm{C} ; \mathrm{CO}=6,2^{\circ} \mathrm{C}$; and $\left.\mathrm{BO}=4,4^{\circ} \mathrm{C}\right)$. The thermal time, in degree-days (DD), varied from 1670.9 to 2060.7 for grapevine cultivars and from 3179.6 to 3762.0 for kiwi cultivars. The higher DD sum for kiwi crop was given by the higher number of days of its vegetative cycle associated to lower $\mathrm{Tb}$ of the genotypes, when compared to the grapevine crop. The phenological subperiod (budburst to maturation) of the fruit trees in $100 \%$ of the assessed cases responded more to thermal time (degree-days) than to chronological time (d) to complete the vegetative cycle.
\end{abstract}

KEYWORDS: growing season, dormancy, degree-days, Actinidia sp., Vitis sp. 


\section{INTRODUÇÃO}

A disponibilidade térmica tem influência direta no crescimento e no desenvolvimento das plantas. Temperaturas elevadas aceleram o metabolismo vegetal, enquanto baixas temperaturas reduzem 0 crescimento e prolongam o ciclo das plantas. Em regiões com oscilação térmica anual acentuada, como no Sul do Brasil, as espécies frutíferas de clima temperado entram em dormência no outono/inverno, retornando a um ciclo vegetativo na primavera, ao se elevarem as temperaturas do ar (BERGAMASCHI 2007).

Durante o período vegetativo, as plantas respondem à temperatura do ar na forma de soma térmica, em graus-dia (SOUZA et al. 2011a, CARDOSO et al. 2012). Este modelo representa a integração das temperaturas efetivas para o crescimento das plantas, fixadas entre dois limites térmicos: as temperaturas base inferior (Tb) e superior (TB) (BERGAMASCHI 2007). O limite inferior (Tb) corresponde a temperatura a partir da qual o metabolismo vegetal é acionado, e o limite superior (TB) corresponde a temperatura máxima permitida para realização da fotossíntese pelas plantas. Embora o modelo de graus-dia atenda a ambos limites térmicos (Tb e TB), desprezando períodos em que a temperatura estiver abaixo de $\mathrm{Tb}$ e acima de $\mathrm{TB}$, a maioria dos modelos considera para o cálculo do tempo térmico somente o limite inferior $(\mathrm{Tb})$ (ARNOLD 1959, VILLA NOVA et al. 1972), quando aplicados às frutíferas nas condições do Sul do Brasil. Isto é devido às temperaturas máximas do dia na região Sul do Brasil dificilmente ultrapassarem a TB das frutíferas temperadas (LAZZARI 2011) não excedendo, portanto, o limite térmico permitido para as plantas realizarem a fotossíntese.

O conceito de tempo térmico ou graus-dia, obtido a partir da temperatura-base das culturas, reduz discrepâncias na caracterização do ciclo das plantas sob diferentes regimes térmicos, se comparada a dias de calendário (DAY et al. 2008). Para uma mesma espécie ou variedade, a duração das etapas fenológicas e do ciclo varia entre anos e locais, dependendo das condições térmicas do ambiente. A necessidade em graus-dia, por outro lado, tende a ser uniforme para um mesmo genótipo, em diferentes ambientes térmicos. Isto facilita a caracterização do padrão fenológico das plantas para diferentes locais e períodos, a menos que outros fatores possam interferir (BERGAMASCHI 2007). A soma de graus-dia que a planta necessita para completar parte ou todo o ciclo tem sido utilizada para caracterizar as fases fenológicas e/ou a produção das plantas (SCHWARTZ 2003). Este parâmetro permite conhecer as exigências térmicas das culturas, e contribui para a definição antecipada das datas fenológicas, indicando o potencial climático da região para produção e permitindo o planejamento e manejo das atividades agrícolas (MILLER et al. 2001).

Em fruteiras de clima temperado, após ter a necessidade em frio suprida para a superação da dormência no outono/inverno, as gemas entram na fase de exigência em calor (ANZANELLO 2012). Para MILLER et al. (2001), cada subperíodo de desenvolvimento da planta tem seu requerimento total de calor, acima da Tb. A data para início do acúmulo de calor é conhecida como "biofix". No caso das frutíferas de clima temperado, esta data é quando ocorre o término da endodormência (dormência controlada pelo frio), em que as plantas param de contabilizar frio e iniciam a computar calor para o alcance da brotação, fase denominada de ecodormência. A data "biofix" também pode ser a data inicial de um subperíodo (ex.: floração à maturação), não importando o quanto de calor foi acumulado nas fases anteriores (LAZZARI 2011).

Os modelos de graus-dia aplicados às frutíferas, baseados na Tb, apresentam algumas limitações. Para a estimativa dos graus-dia considera-se, de modo geral, um único valor de Tb para as fruteiras de clima temperado, que é de $4,5^{\circ} \mathrm{C}$, segundo RICHARDSON et al. (1975). Entretanto, MORLEY-BUNKER \& SALINGER (1987), PEDRO JUNIOR et al. (1994) e NAGATA et al. (2000) determinam a temperatura basal mínima de $10{ }^{\circ} \mathrm{C}$ para as culturas da videira e do quivizeiro. É importante considerar a especificidade, tanto entre espécies quanto entre cultivares de uma mesma espécie frutífera em relação ao parâmetro de Tb, para um melhor ajuste dos modelos de predição da fenologia. De acordo com BERLATO \& SUTILLI (1976), modelos matemáticos podem ser falhos ao não discriminar a Tb de cada espécie ou, classe de genótipo (precoce, intermediário e tardio), como fundamento para modelagem precisa da fenologia das plantas.

PUTTI et al. (2003) e ANZANELLO (2012) relatam a possibilidade de existir diferenças na Tb entre cultivares, contrastantes em exigência térmica, para uma mesma espécie frutífera. Isto porque observaram que cultivares de macieiras contrastantes em necessidade térmica na dormência mostram diferentes intervalos de temperaturas efetivas de frio, sendo maior $\left(3\right.$ a $\left.12{ }^{\circ} \mathrm{C}\right)$ para genótipos de baixa demanda de frio hibernal, e menor $\left(3 \mathrm{a} 6^{\circ} \mathrm{C}\right)$ para àqueles com alta necessidade de frio. Com isso, sugerem que a Tb, a partir da qual é computado calor pelas plantas, pode-se diferir entre cultivares contrastantes em exigência térmica. Tal distinção resultaria em diferenças na necessidade de calor nos subperíodos fenológicos do ciclo vegetativo entre genótipos, se comparada à soma térmica calculada pela Tb padrão das culturas. 
A definição da Tb, aplicada às frutíferas, também está limitada por não considerar as respostas biológicas das plantas. Tal parâmetro deveria ser determinado por cunho experimental, ponderando a tradução biológica das plantas, e não reportar-se exclusivamente à modelagem estatística, como àquela sugerida por RICHARDSON et al. (1975), SPIEGEL-ROY \& ALSTON (1979), HIDALGO (1980), MORLEYBUNKER \& SALINGER (1987), PEDRO JUNIOR et al. (1994), RODRÍGUEZ (1995), NAGATA et al. (2000), MARRA et al. (2002), GREEN (2007) e SOUZA et al. (2011b). Para estes, a Tb foi resultante de uma estimativa, e não de uma observação real obtida experimentalmente, ou seja, a Tb é definida a partir do resultado de menor desvio-padrão que uma temperatura, escolhida a priori, confere à soma térmica de uma série de anos/experimentos avaliados (ARNOLD 1959). O uso de métodos biológicos, além de representar as condições intrínsecas das plantas, permite isolar fatores ambientais, como os efeitos da temperatura do ar sobre as plantas (ANZANELLO et al. 2014).

O objetivo deste trabalho foi determinar a temperatura base inferior, soma térmica e fenologia de cultivares de videira e de quivizeiro cultivadas na região Sul do Brasil.

\section{MATERIAL E MÉTODOS}

\section{Tb inferior das frutíferas de clima temperado}

Estacas de ano de espécies frutíferas de clima temperado foram coletadas no período hibernal de 2015. As espécies e cultivares selecionadas foram: videira (Chardonnay, Concord, Niágara Branca, Isabel e Bordô) e quivizeiro (Bruno, Elmwood, Monty, MG06 e Yellow Queen). As estacas das cultivares de quivizeiro foram coletadas em pomares experimentais pertencentes ao Departamento de Diagnóstico e Pesquisa Agropecuária (DDPA) da Secretaria da Agricultura, Pecuária e Irrigação (SEAPI) do Estado do $\mathrm{RS}$, em Veranópolis, RS, e as estacas das cultivares de videira foram coletadas em vinhedos comerciais no mesmo município.

As estacas foram amostradas com 25 a $35 \mathrm{~cm}$ de comprimento, apresentando em média seis gemas para as cultivares de videira e quivizeiro. A amostragem foi realizada no dia 03 de junho de 2015 para todas as cultivares, com zero horas de frio $\leq 7,2{ }^{\circ} \mathrm{C}$ (HF) a campo. Na seleção do material para coleta foram consideradas a maturidade das gemas (gemas bem fechadas), a sanidade e o vigor das estacas, priorizando aquelas com crescimento intermediário.

Após coletadas no campo, as estacas foram enroladas em feixes, umedecidas e armazenadas em sacos plásticos até o início do processamento. Em seguida, o material foi desinfestado, passando por um processo de limpeza composto sequencialmente de etanol $70 \%$ por $45-60 \mathrm{~s}$, hipoclorito de sódio a 2,5\% por 20 min e três enxágues com água destilada. Após, as estacas foram secas à sombra por cerca de $30 \mathrm{~min}$ (ANZANELLO et al. 2014).

As estacas intactas foram organizadas em feixes de 20-25 unidades cada, embaladas com filme plástico preto de polietileno, e submetidas a um período prolongado de frio $\left(1.008 \mathrm{HF}\right.$ a $\left.0{ }^{\circ} \mathrm{C}\right)$ sem fotoperíodo, em câmaras incubadoras climatizadas marca Eletrolab, modelo EL202, para a superação da endodormência.

Após o período de frio, as estacas foram processadas pelo método biológico de estacas de nós isolados (ANZANELLO et al. 2014). As estacas foram fragmentadas em segmentos de $7 \mathrm{~cm}$ de comprimento, contendo apenas uma gema lateral na porção superior. Em seguida, as estacas foram plantadas em espuma fenólica umedecida, acondicionada em potes plásticos. Os potes com as estacas foram submetidos a cinco níveis de calor $\left(4,6,8,10\right.$ e $\left.12{ }^{\circ} \mathrm{C}\right)$, para determinação da temperatura efetiva de calor, em câmaras incubadoras, por um período de 150 dias, sem a presença de fotoperíodo. Para cada tratamento de calor se dispôs de três repetições (três potes com 10 estacas). As câmaras incubadoras foram subdivididas em três prateleiras, sendo cada repetição acondicionada numa prateleira. Isto visou controlar possíveis diferenças de circulação de ar no interior das câmaras incubadoras.

Ao longo do período de avaliação (150 dias), as gemas foram analisadas a cada 2 a 3 dias, anotandose a data de brotação de cada gema em estádio de ponta verde (CARVALHO et al. 2010). Com os dados de brotação, processaram-se as variáveis de tempo médio para brotação das gemas, que representou o número médio de dias passados entre a instalação do experimento e a detecção do estádio de ponta verde; e a taxa final de brotação, que representou a porcentagem de estacas com gemas que atingiram o estádio de ponta verde (CARVALHO \& BIASI 2012).

A irrigação das estacas nas câmaras incubadoras foi realizada adicionando-se em torno de $20 \mathrm{ml}$ de água por pote a cada 48-72 horas. A reposição de água foi suficiente para saturar a espuma fenólica, evitando-se acúmulo de água livre no pote. Durante o período de indução e avaliação da brotação, preveniu-se o surgimento de doenças pela utilização de defensivos químicos a base de pirimetamil e 
tebuconazol (sistêmicos) e iprodiona e captan (contato), pulverizados na dosagem de 1,5 a 2,0 ml $\mathrm{L}^{-1}$, exceto aquele à base de tebuconazol, cuja dosagem foi de $1,0 \mathrm{ml} \mathrm{L}^{-1}$. A aplicação foi realizada a cada 14 a 21 dias, intercalando-se os produtos de contato e sistêmico.

\section{Base de dados fenológicos}

Uma base de dados fenológicos das cultivares estudadas foi organizada para, associada aos dados meteorológicos correspondentes, quantificar a soma térmica (graus-dia) durante o ciclo vegetativo das frutíferas. Utilizou-se a Tb encontrada para cada genótipo para o cálculo da soma térmica. A base de dados fenológicos foi composta pelas datas de brotação ( $50 \%$ de gemas brotadas), início da floração (10\% de flores abertas), final da floração ( $100 \%$ de pétalas caídas) e final de maturação (data de colheita dos frutos). Os dados foram extraídos de cadernos de campo, coletados em pomares experimentais (tipo coleção), do DDPA-SEAPI (para as cultivares de quivizeiro) e da EMBRAPA Uva e Vinho (para as cultivares de videira). Os registros de fenologia compreenderam as seguintes faixas temporais: para as videiras 'Chardonnay', 'Concord' e 'Bordô' de 1984 a 1993; 'Isabel' de 1988 a 1997; 'Niágara Branca' de 1989 a 1998 ; e para os quivizeiros 'Bruno', 'Monty', 'Elmwood', 'MG06' e 'Yellow Queen' de 1997 a 2006.

\section{Base de dados meteorológicos}

Foram utilizados dados diários de temperatura mínima (Tm) e máxima do ar (TM), registrados pela Estação Meteorológica do DDPA-SEAPI de Veranópolis, RS, e da EMBRAPA Uva e Vinho de Bento Gonçalves, RS. O período de coleta dos dados meteorológicos correspondeu ao igual período de coleta dos dados fenológicos, sendo utilizados os dados meteorológicos da Estação de Veranópolis para confrontar com os dados fenológicos de quivizeiro e os dados meteorológicos da Estação de Bento Gonçalves para confrontar com os dados fenológicos da cultura da videira.

\section{Soma térmica (graus-dia)}

Para o cálculo de graus-dia, atenderam-se os seguintes subperíodos fenológicos para as culturas da videira e do quivizeiro: da brotação ao início da floração; do início da floração ao final da floração; do final da floração ao final da maturação; e da brotação ao final da maturação. Os respectivos intervalos tiveram um acompanhamento com base no acúmulo de graus-dia, segundo metodologia proposta por VILLA NOVA et al. (1972).

$$
\begin{aligned}
& \text { GD }=(T m-T b)+(T M-T m) / 2, \text { para } T m>T b \\
& G D=(T M-T b)^{2} / 2(T M-T m), \text { para } T m<T b \\
& G D=0, \text { para } T b>T M .
\end{aligned}
$$

Sendo: GD os graus-dia; TM a temperatura máxima diária $\left({ }^{\circ} \mathrm{C}\right)$; Tm a temperatura mínima diária $\left({ }^{\circ} \mathrm{C}\right)$ e Tb a temperatura base inferior $\left({ }^{\circ} \mathrm{C}\right)$.

A soma térmica necessária para o alcance dos subperíodos fenológicos foi relacionada com a duração média das respectivas etapas, em dias, para comparar medidas distintas de acompanhamento do ciclo fenológico das espécies frutíferas.

\section{Análise estatística}

Os dados referentes à taxa de brotação final foram submetidos à análise de variância. Os resultados com diferenças significativas, pelo teste $\mathrm{F}$, tiveram suas médias submetidas ao teste de Tukey, ao nível de significância de $5 \%$ de probabilidade. Os dados do inverso do número de dias para brotação (1/dias para brotação) foram inseridos em curvas de regressão para definição da Tb das cultivares frutíferas. Para as variáveis relacionadas à soma térmica e dias, computadas durante os subperíodos fenológicos do ciclo das frutíferas, a análise foi realizada por estatística descritiva, considerando medidas de tendência central (média) e medidas de dispersão (coeficiente de variação).

\section{RESULTADOS E DISCUSSÃO}

Para a avaliação da Tb das cultivares frutíferas de clima temperado, são mostrados nas Figuras 1 e 2 os resultados de porcentagem de brotação e do inverso do número de dias para brotação das gemas, quando submetidas às temperaturas de $4,6,8,10$ e $12^{\circ} \mathrm{C}$, após um período prolongado de frio de $1.008 \mathrm{HF}$ a $0^{\circ} \mathrm{C}$.

Nas temperaturas que induziram a brotação das gemas, os percentuais obtidos foram próximos a $100 \%$ para todas as cultivares. Para as temperaturas consideradas abaixo do limiar de indução do crescimento das gemas, a brotação foi nula, mesmo após 150 dias de exposição. O inverso do número de dias para brotação (1/dias) integrada às diferentes temperaturas resultou em gráficos de regressão linear para todas as cultivares, sendo a Tb dos genótipos definida na interseção da reta ao eixo x. Pela equação da reta, para o inverso do número de dias para brotação igual a zero, obteve-se a Tb, conforme metodologia adotada por PUTTI et al. (2000). Para tais autores, o inverso do número de dias para brotação igual à zero 
está relacionado ao marco inicial, a partir do qual, as plantas retomam o índice de desenvolvimento.

Para LAW \& KELTON (2000), fenômenos naturais aplicados a uma variável dependente de escala achatada, se ajustam adequadamente às funções lineares. Diante disso, a regressão linear é um bom modelo para ajuste aos dados de brotação obtidos no presente estudo, cujos resultados foram alcançados numa faixa comprimida, de 4 a $12{ }^{\circ} \mathrm{C}$. Entretanto, tal regressão não deve ser extrapolada para uso com temperaturas extremas (acima ou abaixo do intervalo de estudo), uma vez que normalmente os processos biológicos não seguem funções lineares. Suas respostas às condições do ambiente e a própria evolução dos fenômenos, no tempo, são tipicamente não lineares. Assim sendo, em geral, os modelos lineares representam apenas simplificações dos processos biológicos (BONHOMME 2000).

Para as videiras, 'Chardonnay', 'Niágara Branca', 'Isabel' e 'Bordô' apresentaram a Tb inferior na faixa de 4 a $6{ }^{\circ} \mathrm{C}$ (Figura 1). Já, a 'Concord' apresentou Tb inferior na faixa de 6 a 8 ํ. Pela equação da regressão linear, obteve-se o valor de temperatura mínima basal de $4,2{ }^{\circ} \mathrm{C}$ para 'Chardonnay', 4,3 ${ }^{\circ} \mathrm{C}$ para 'Isabel', 4,1 ${ }^{\circ} \mathrm{C}$ para 'Niágara Branca', 4,4 ${ }^{\circ} \mathrm{C}$ para 'Bordô' e 6,2 ${ }^{\circ} \mathrm{C}$ para 'Concord'. Os resultados de Tb das cultivares aproximam-se dos obtidos por RICHARDSON et al. (1975) de $4,5{ }^{\circ} \mathrm{C}$ (exceto para a 'Concord'), mas contrariam aqueles alcançados por HIDALGO (1980), PEDRO JUNIOR et al. (1994) e NAGATA et al. (2000), que definem a temperatura basal mínima de $10^{\circ} \mathrm{C}$ para a cultura da videira.

Para as cultivares de quivizeiro, a Tb inferior de 'Bruno', 'Monty', 'Elmwood', 'MG06' e 'Yellow Queen' enquadraram-se na faixa de 2 a $4{ }^{\circ} \mathrm{C}$ (Figura 2). Pela equação linear, obteve-se o valor da temperatura mínima basal de 3,0 ${ }^{\circ} \mathrm{C}$ para 'Bruno' e 'Yellow Queen', de 3,3 ${ }^{\circ} \mathrm{C}$ para 'Monty', de 3,1 ${ }^{\circ} \mathrm{C}$ para 'Elmwood' e de 3,2 ${ }^{\circ} \mathrm{C}$ 'MG06'. Para o quivizeiro, MORLEY-BUNKER \& SALINGER (1987) estimaram em 7,5 ${ }^{\circ} \mathrm{C}$ a temperatura mínima basal e GREEN (2007) definiram em $10^{\circ} \mathrm{C}$ a temperatura abaixo da qual o crescimento da cultura é desprezível ou nulo, valores que se opõem aqueles encontrados no presente estudo, assim como ao dado referencial de $4,5^{\circ} \mathrm{C}$ proposto por RICHARDSON et al. (1975).

Os resultados de $\mathrm{Tb}$ do presente estudo se destacam pela $\mathrm{Tb}$ diferir entre espécies frutíferas, configurando a inexistência de um único limiar térmico inferior para o crescimento das frutíferas de clima temperado, como a defendida de $4,5{ }^{\circ} \mathrm{C}$ e amplamente empregada para as fruteiras temperadas (RICHARDSON et al. 1975, BERGAMASCHI 2007, CARDOSO et al. 2012).

Para HAWERROTH et al. (2010), o uso de métodos biológicos é imprescindível para possibilitar o teste isolado de fatores ambientais. Em condições de campo, pela restrição de controle (ex.: radiação, precipitação e temperatura), não há possibilidade de se determinar com precisão a Tb das espécies frutíferas, devido à complexidade de interações entre o ambiente e a planta. Por tomar dados obtidos a partir de um teste biológico, conduzido em condições controladas, os resultados apresentados nas Figuras 1 e 2 representam fielmente as respostas das plantas à variável de temperatura do ar, isolando os demais fatores. Os resultados contrapõem-se àqueles descritos por RICHARDSON et al. (1975), SPIEGEL-ROY \& ALSTON (1979), HIDALGO (1980), MORLEY-BUNKER \& SALINGER (1987), PEDRO JUNIOR et al. (1994), RODRÍGUEZ (1995), NAGATA et al. (2000), MARRA et al. (2002), GREEN (2007) e SOUZA et al. (2011b), que determinaram a Tb por simulação usando, unicamente a modelagem estatística proposta por ARNOLD (1959).

Os registros de fenologia, a partir da série histórica observada, indicaram que para as cultivares de videira a 'Chadornnay' foi a mais precoce e a 'Isabel' a mais tardia, considerando as datas de brotação e de final da maturação (Tabela 1). Tais dados confirmam aqueles descritos por GIOVANNINI (2008), para a cultura da videira. Para as cultivares de quiziveiro, 'Yellow Queen' e 'MG06' foram as mais precoces se comparadas aos demais genótipos, com base na data de fim da maturação (Tabela 1). Conforme SILVEIRA et al. (2012), cultivares de quivizeiro pertencentes a espécie Actinidia chinnensis Planch (Yellow Queen e MG06) necessitam de menor número de horas de frio durante o período de dormência conferindo maior precocidade de produção se comparada às cultivares do gênero Actinidia deliciosa A. Chev (Bruno, Elmwood e Monty).

A soma de graus-dia do subperíodo fenológico compreendido entre o início do ciclo vegetativo ao final da maturação, ou seja, da brotação ao final da maturação variou de 1670,9 a 2060,7 GD para as cultivares de videira (1809,4 GD para 'Chardonnay', 2060,7 GD para 'Isabel', 1792,8 GD para 'Niágara Branca', 1670,9 GD para 'Concord' e 1802,4 GD para 'Bordô') e de 3179,6 a 3762,0 GD para as cultivares de quivizeiro (3760,3 GD para 'Bruno', 3762,0 GD para 'Monty', 3711,3 GD para 'Elmwood', 3179,6 GD para 'MG06' e 3336,3 GD para 'Yellow Queen') (Tabela 2). A maior soma de graus-dia para a cultura do quivizeiro é dada pelo maior número de dias do seu ciclo vegetativo associada a menor Tb dos genótipos, se comparada à cultura da videira (Figuras 1 e 2, Tabela 2). 


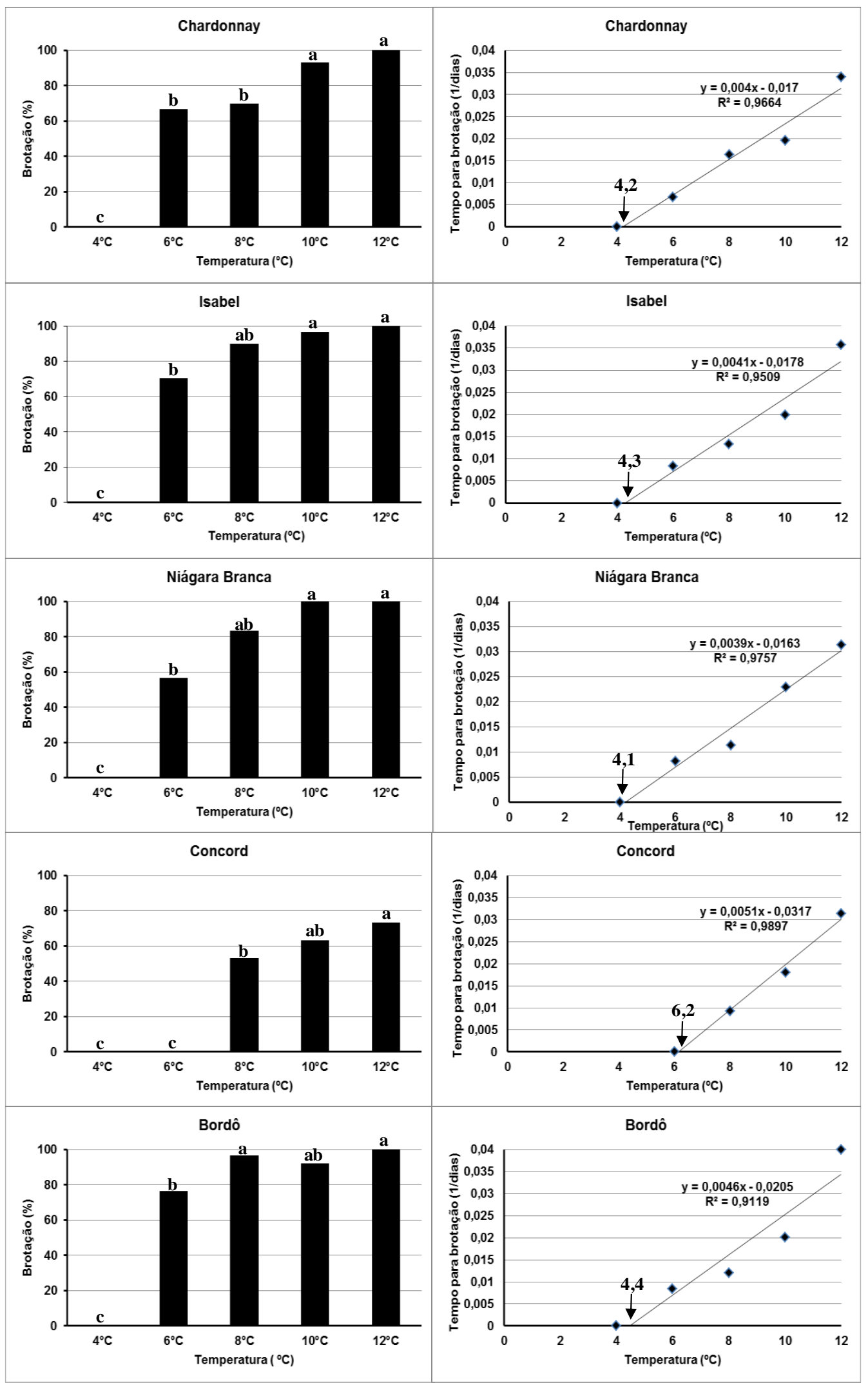

Figura 1. Brotação total (gráficos à esquerda) e regressões lineares (gráficos à direita) utilizadas para definição da Tb das videiras 'Chardonnay', 'Isabel', 'Niágara Branca', 'Concord' e 'Bordô'. As gemas foram submetidas às temperaturas de $4,6,8,10$ e $12{ }^{\circ} \mathrm{C}$ por 150 dias, após $1.008 \mathrm{HF}$ a temperatura de $0{ }^{\circ} \mathrm{C}$. Veranópolis, RS. 2015. Médias seguidas pela mesma letra não diferem pelo teste de Tukey a $5 \%$.

Figure 1. Total budburst (graphics to the right) and linear regressions (graphics to the left) used to define Tb of grapevines 'Chardonnay', 'Isabel', 'Niágara Branca', 'Concord' and 'Bordô'. The buds were subjected to temperatures of $4,6,8,10$ and $12^{\circ} \mathrm{C}$ for $150 \mathrm{~d}$, after $1,008 \mathrm{HC}$ at $0^{\circ} \mathrm{C}$. Veranópolis, RS. 2015. Means followed by the same letter do not differ from Tukey's test at $5 \%$ probability. 

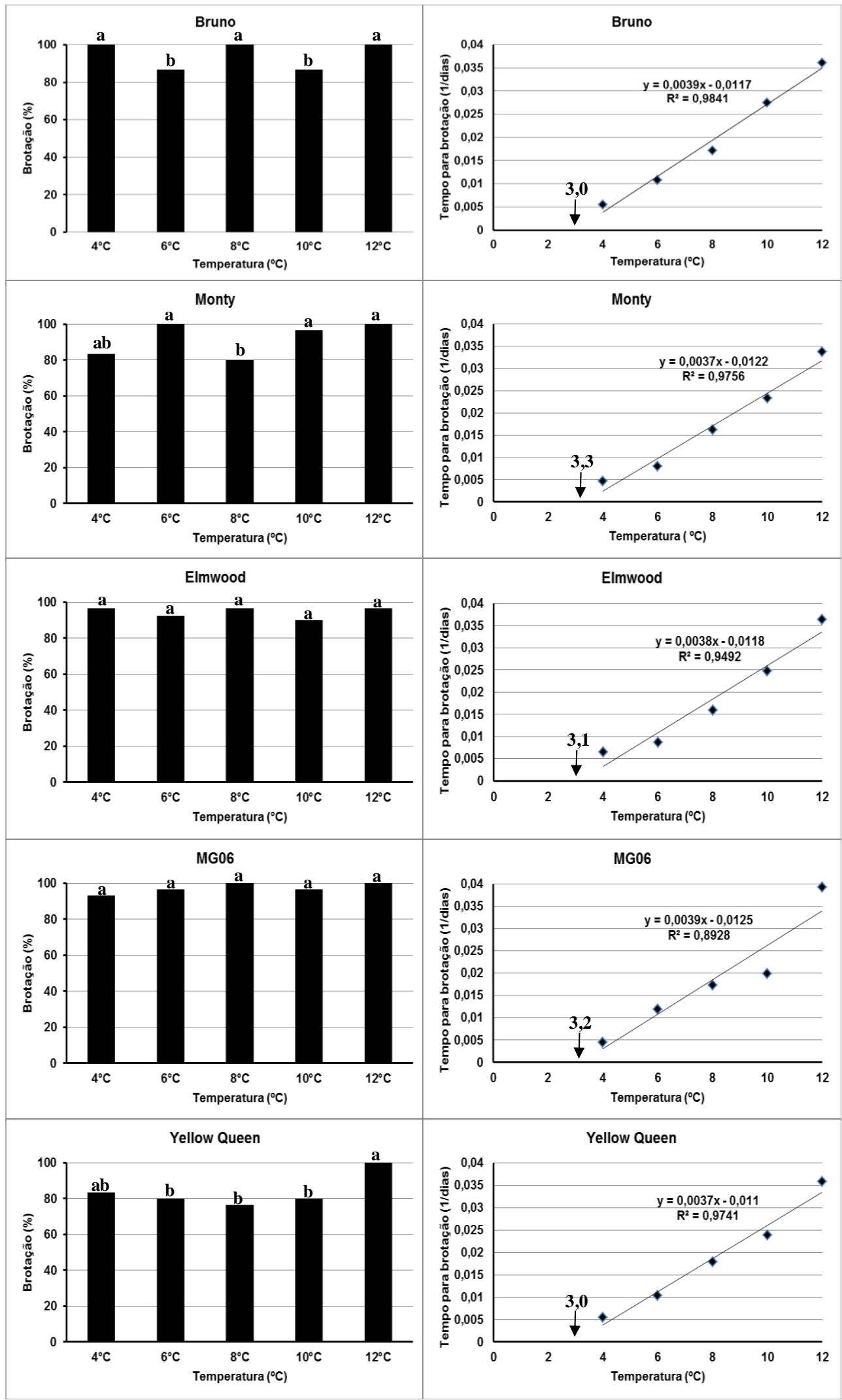

Figura 2. Brotação total (gráficos à esquerda) e regressões lineares (gráficos à direita) utilizadas para definição da Tb dos quivizeiros 'Bruno', 'Monty', 'Elmwood', 'MG06' e 'Yellow Queen'. As gemas foram submetidas às temperaturas de $4,6,8,10$ e $12{ }^{\circ} \mathrm{C}$ por 150 dias, após $1.008 \mathrm{HF}$ a temperatura de $0{ }^{\circ} \mathrm{C}$. Veranópolis, RS. 2015. Médias seguidas pela mesma letra não diferem pelo teste de Tukey a $5 \%$.

Figure 2. Total budburst (graphics to the right) and linear regressions (graphics to the left) used to define $T b$ of kiwis 'Bruno', 'Monty', 'Elmwood', 'MG06' and 'Yellow Quenn'. The buds were subjected to temperatures of $4,6,8,10$ and $12^{\circ} \mathrm{C}$ for $150 \mathrm{~d}$, after 1,008 $\mathrm{HC}$ at $0^{\circ} \mathrm{C}$. Veranópolis, RS. 2015. Means followed by the same letter do not differ from Tukey's test at $5 \%$ probability. 
Tabela 1. Datas médias de fenologia das cultivares de videira Chardonnay, Isabel, Niágara Branca, Concord e Bordô, e de quivizeiro Bruno, Monty, Elmwood, MG06 e Yellow Queen considerando um período de 10 anos de avaliação, em pomares experimentais do DDPA-SEAPI, Veranópolis, RS, e da Embrapa Uva e Vinho, Bento Gonçalves, RS.

Table 1. Mean phenological dates (Day/Month format) of cultivars of grapevine Chardonnay, Isabel, Niágara Branca, Concord and Bordô, and of kiwi Bruno, Monty, Elmwood, MG06 e Yellow Queen, considering a 10-year evaluation period, in experimental orchards at DDPA-SEAPI, Veranópolis, RS, and the Embrapa Uva e Vinho, Bento Gonçalves, RS.

\begin{tabular}{|c|c|c|c|c|}
\hline \multicolumn{5}{|c|}{ Datas Fenológicas } \\
\hline Cultivar & Brotação & Início & Fim & Final Maturação \\
\hline Chardonnay & $08 / 09$ & $16 / 10$ & $03 / 11$ & $08 / 01$ \\
\hline Isabel & $24 / 09$ & $22 / 10$ & $03 / 11$ & $21 / 02$ \\
\hline Niágara Branca & $19 / 09$ & $07 / 10$ & $22 / 10$ & $24 / 01$ \\
\hline Concord & $26 / 09$ & $20 / 10$ & $03 / 11$ & $20 / 01$ \\
\hline Bordô & $21 / 09$ & $16 / 10$ & $29 / 10$ & $15 / 01$ \\
\hline \multicolumn{5}{|c|}{ Datas Fenológicas } \\
\hline Cultivar & Brotação & Iníni & $\Gamma$ & Final Maturação \\
\hline Bruno & $16 / 09$ & $20 / 10$ & $16 / 11$ & $20 / 04$ \\
\hline Monty & $16 / 09$ & $21 / 10$ & $18 / 11$ & $13 / 05$ \\
\hline Elmwood & $18 / 10$ & $23 / 10$ & $30 / 11$ & $11 / 05$ \\
\hline MG06 & $12 / 09$ & $07 / 10$ & $24 / 10$ & $08 / 03$ \\
\hline Yellow Queen & $20 / 09$ & $17 / 10$ & $17 / 11$ & $20 / 03$ \\
\hline
\end{tabular}

Considerando as especificidades entre cultivares, a videira 'Concord' apresentou menor número de GD em função da sua maior Tb inferior em relação aos demais genótipos (Figura 1, Tabela 2). Para a cultivar Isabel, o maior número de GD e dias de calendário deve-se ao período mais longo compreendido entre o subperíodo fenológico do final da floração ao final da maturação (Tabela 2). Para as cultivares de quivizeiro, a maior precocidade dos genótipos MG06 e Yellow Queen é expressa em função do menor GD e dias de calendário entre o final da floração ao final da maturação, se comparada as demais variedades (Tabela 2).

Tabela 2. Soma térmica média, em graus-dia (GD), e duração média, em dias, para os subperíodos fenológicos do ciclo vegetativo de videiras 'Chardonnay', 'Isabel', 'Niágara Branca', 'Concord' e 'Bordô', e de quivizeiro 'Bruno', 'Monty', 'Elmwood' 'MG06' e 'Yellow Queen' considerando um período de 10 anos de avaliação.

Table 2. Mean thermal time, in degree-days (DD), and mean duration, in days, of the phenological subperiods of the growing season of grapevines 'Chardonnay', 'Isabel', 'Niágara Branca', 'Concord' and 'Bordô', and of kiwis 'Bruno', 'Monty', 'Elmwood', 'MG06' e 'Yellow Queen', over a 10-year evaluation period.

\begin{tabular}{lcccccccc}
\hline & \multicolumn{9}{c}{ Subperíodos fenológicos } & \multicolumn{2}{c}{ FF - M } & \multicolumn{2}{c}{ B - FM } \\
\multicolumn{1}{c}{ Cultivar } & GD $-\mathrm{IF}^{*}$ & Dias & GD & Dias & GD & Dias & GD & Dias \\
\hline Chardonnay & 420,1 & 35 & 302,5 & 22 & 1086,7 & 67 & 1809,4 & 125 \\
Isabel & 366,7 & 29 & 170,5 & 12 & 1523,4 & 111 & 2060,7 & 152 \\
N. Branca & 250,3 & 20 & 192,7 & 19 & 1349,9 & 93 & 1792,8 & 132 \\
Concord & 336,0 & 30 & 170,9 & 18 & 1164,0 & 79 & 1670,9 & 128 \\
Bordô & 353,1 & 29 & 174,4 & 14 & 1274,9 & 77 & 1802,4 & 121 \\
\hline
\end{tabular}

\begin{tabular}{lcccccccc}
\hline & \multicolumn{9}{c}{ Subperíodos fenológicos } & \multicolumn{2}{c}{ FF - M } & \multicolumn{2}{c}{ B - FM } \\
\multicolumn{1}{c}{ Cultivar } & GD - IF & Dias & GD & Dias & GD & Dias & GD & Dias \\
\hline Bruno & 626,4 & 41 & 317,4 & 20 & 2816,6 & 186 & 3760,3 & 247 \\
Monty & 401,6 & 35 & 270,8 & 18 & 3089,6 & 162 & 3762,0 & 215 \\
Elmwood & 423,8 & 32 & 269,0 & 21 & 3018,8 & 177 & 3711,6 & 230 \\
MG06 & 319,7 & 25 & 230,1 & 17 & 2629,8 & 123 & 3179,6 & 165 \\
Y. Queen & 384,9 & 33 & 336,1 & 24 & 2615,3 & 135 & 3336,3 & 192 \\
CV (\%) & --- & --- & --- & --- & --- & --- & & \\
\hline
\end{tabular}

$\left(^{*}\right)$ B: brotação, IF: início da floração, FF: fim da floração, FM: final da maturação. 
$\mathrm{Na}$ comparação entre dias de calendário versus soma térmica (graus-dia) durante o subperíodo fenológico da brotação ao final da maturação das cultivares estudadas, em $100 \%$ dos casos, a soma térmica (graus-dia) conferiu menor coeficiente de variação em relação aos dias do calendário (Tabela 3). Pela redução na variabilidade observada, sob análise da série histórica dos anos observados, reforça-se a hipótese de que a fenologia das plantas responde mais ao "tempo térmico" (graus-dia) do que ao tempo cronológico (dias), para o acompanhamento do ciclo vegetativo das plantas.

Tabela 3. Média e coeficiente de variação (CV) entre graus-dia e dias de calendário para o subperíodo fenológico brotação ao final da maturação de videiras 'Chardonnay', 'Isabel', ' Niágara Branca', 'Concord' e 'Bordô', e de quivizeiro 'Bruno', 'Monty', 'Elmwood', 'MG06' e 'Yellow Queen' considerando um período de 10 anos de avaliação.

Table 3. Mean and coefficient of variation (CV) between degree-days and calendar days for the phenological subperiod budburst at the end of the maturation of grapevines 'Chardonnay', 'Isabel', 'Niágara Branca', 'Concord' and 'Bordô', and of kiwis 'Bruno', 'Monty', 'Elmwood', 'MG06' e 'Yellow Queen', over a 10-year evaluation period.

\begin{tabular}{|c|c|c|c|c|c|}
\hline \multirow{2}{*}{\multicolumn{2}{|c|}{ Cultura/Cultivar }} & \multicolumn{4}{|c|}{ Subperíodo fenológico: Brotação - Final da Maturação } \\
\hline & & \multicolumn{2}{|c|}{ Graus-dia } & \multicolumn{2}{|c|}{ Dias de calendário } \\
\hline & & Média & CV (\%) & Média & $\mathrm{CV}(\%)$ \\
\hline \multirow[t]{5}{*}{ Videira } & Chardonnay & 1809,4 & 11,2 & 125 & 19,3 \\
\hline & Isabel & 2060,7 & 9,3 & 152 & 17,5 \\
\hline & Niágara Branca & 1792,8 & 12,5 & 132 & 23,6 \\
\hline & Concord & 1670,9 & 13,5 & 128 & 20,8 \\
\hline & Bordô & 1802,4 & 9,4 & 121 & 22,6 \\
\hline \multirow[t]{5}{*}{ Quivizeiro } & Bruno & 3760,3 & 11,3 & 247 & 25,0 \\
\hline & Monty & 3762,0 & 12,7 & 215 & 27,4 \\
\hline & Elmwood & 3711,6 & 10,7 & 230 & 18,5 \\
\hline & MG06 & 3179,6 & 16,2 & 165 & 20,2 \\
\hline & Yellow Queen & 3336,3 & 11,6 & 192 & 19,6 \\
\hline
\end{tabular}

Para BERGAMASCHI (2007), o uso do número de dias de calendário como variável independente, ao invés do critério de tempo térmico (graus-dia), restringe a validade dos modelos de simulação a ambientes semelhantes àqueles em que os dados foram gerados. Haverá discrepâncias de estimativas sempre que o modelo for utilizado em locais ou épocas com condições térmicas diferentes. Para CARDOSO et al. (2012) o uso da soma de graus-dia, baseada no acúmulo energético acima de determinada $\mathrm{Tb}$, é de uso abrangente na simulação do desenvolvimento fenológico e do crescimento das plantas. Embora a água e o fotoperíodo também possam influenciar a fenologia das plantas, na maioria das culturas a soma térmica tem relação direta com o desenvolvimento das plantas (MILLER et al. 2001). A predição de períodos críticos, as exigências ecoclimáticas das culturas, a programação de práticas de manejo importantes, e o emprego da modelagem em zoneamentos agroclimáticos, são alguns exemplos de aplicações dos graus-dia em cultivos agrícolas (DAY et al. 2008). Para SOUZA et al. (2011a), a soma de graus-dia permite estimar com precisão a duração do ciclo e a ocorrência de fases ou eventos importantes ao longo da evolução fenológica das plantas, conforme demostrado na Tabela 2.

A caracterização da $\mathrm{Tb}$ e a estimativa da fenologia a partir do acúmulo de soma térmica tem importância frente aos cenários de mudanças climáticas. O aquecimento global tende a elevar a temperatura do ar e causar alterações no acúmulo de graus-dia e, consequentemente, mudando a época de ocorrência das etapas fenológicas (LEGAVE et al. 2008). De modo geral, espera-se um adiantamento do ciclo vegetativo devido às culturas acumularem mais soma térmica diária, acima da $\mathrm{Tb}$, pela elevação da temperatura média do ar. Os impactos deste trabalho, com a definição da Tb dos genótipos, poderão servir para prever alterações nos padrões de fenologia advindos das mudanças climáticas e, predizendo, inclusive, suas implicações agronômicas.

\section{CONCLUSÃO}

A temperatura base inferior difere entre espécies frutíferas de clima temperado, sendo maior para a videira e menor para o quivizeiro. A fenologia das plantas frutíferas responde mais ao tempo térmico do que ao tempo cronológico, ou seja, é mais dependente da soma térmica (graus-dia) do que a dias de calendário para completar o seu ciclo. A diferenciação da temperatura base entre genótipos é um importante fator a ser considerado para ajustes de modelos de fenologia, como fundamento para melhoria da sua precisão e aplicabilidade na agricultura. 


\section{REFERÊNCIAS}

ANZANELLO R. 2012. Fisiologia e modelagem da dormência de gemas em macieira. Tese (Doutorado em Fitotecnia). Porto Alegre: UFRGS. 281p.

ANZANELLO R et al. 2014. Métodos biológicos para avaliação da brotação de gemas em macieira para modelagem da dormência. Semina: Ciências Agrárias 35: 1163-1176.

ARNOLD CY. 1959. The determination and significance of the base temperature in linear heat unit system. Journal of the American Society for Horticultural Science 74: 430-445.

BERGAMASCHI H. 2007. O clima como fator determinante da fenologia das plantas. In: REGO GM et al. (Ed.). Fenologia ferramenta para conservação, melhoramento e manejo de recursos vegetais arbóreos. Colombo: Embrapa Florestas. p. 291-310.

BERLATO MA \& SUTILI VR. 1976. Determinação das temperaturas-base dos subperíodos emergência-pendoamento e emergência-espigamento de três cultivares de milho (Zea mays L.). In: Reunião Técnica do Milho e Sorgo, 21. Anais... Porto Alegre: UFRGS. p. 523-527.

BONHOMME R. 2000. Bases and limits to using "degrees day" units. European Journal of Agronomy 13: 1-10.

CARDOSO LS et al. 2012. Disponibilidades climáticas para macieira na região de Vacaria, RS. Ciência Rural 42: 1960 1967.

CARVALHO RIN et al. 2010. Estádios de brotação de gemas de fruteiras de clima temperado para o teste biológico de avaliação de dormência. Revista Acadêmica de Ciências Agrárias e Ambientais 8: 93-100.

CARVALHO RIN \& BIASI LA. 2012. Índice para a avaliação da intensidade de dormência de gemas de fruteiras de clima temperado. Revista Brasileira de Fruticultura 34: 936-940.

DAY $\mathrm{K}$ et al. 2008. Using growing degree hours accumulated thirty days after bloom to predict peach and nectarine harvest date. Acta Horticulturae 803: 163-166.

GIOVANINNI E. 2008. Produção de uvas para vinhos, suco e mesa. 3.ed. Porto Alegre: Renascença. 362p.

GREEN SR. 2007. Kiwifruit dry matter platform: exploiting water stress using summer deficit irrigation. HortResearch 36 : 1-2.

HAWERROTH FJ et al. 2010. Dormência em frutíferas de clima temperado. Pelotas: Embrapa Clima Temperado. 56p. (Documentos 310).

HIDALGO L. 1980. Caracterización microfisica del ecosistema médio-planta em los viñedos españoles. 1.ed. Madrid: Instituto Nacional de Investigaciones Agrárias. Serie producción vegetal 29. 255p.

LAZZARI M. 2011. Cima e fenologia de cultivares de pessegueiro (Prunus persica) na região do Alto e Médio Vale do Uruguai, RS. Tese (Doutorado em Fitotecnia). Porto Alegre: UFRGS. 169p.

LAW ML \& KELTON WD. 2000. Simulation Modeling and Analysis. 3.ed. Boston: McGraw-Hill. 760p.

LEGAVE JM et al. 2008. Selecting models of apple flowering time and understanding how global warming has had an impact on this trait. Journal of Horticultural Science and Biotechnology 83: 76-84.

MARRA FP et al. 2002. Thermal time requirement and harvest time forecast for peach cultivars with different fruit development periods. Acta Horticulturae 592: 523-529.

MILLER P et al. 2001. Using growing degree days to predict plant stages. 1.ed. Bozeman: State University Montana. 8p.

MORLEY-BUNKER MJ \& SALINGER MJ. 1987. Kiwifruit development: the effect of temperature on bud burst and flowering. Weather and Climate 7: 26-30.

NAGATA RK et al. 2000. Temperatura-base e soma térmica (graus-dia) para videiras 'Brasil' e 'Benitaka'. Revista Brasileira de Fruticultura 22: 329-333.

PEDRO JUNIOR MJ et al. 1994. Determinação da Temperatura-base, graus-dia e índice biometeorológico para a videira 'Niagara Rosada'. Revista Brasileira de Agrometeorologia 2: 51-56.

PUTTI GL et al. 2000. Unidades de frio e de calor para a brotação de macieira (Malus domestica, Borkh), "Gala" e "Fuji". Revista Brasileira de Agrociência 6: 194-196.

PUTTI GL et al. 2003. Temperaturas efetivas para a dormência da macieira (Malus domestica Borkh). Revista Brasileira de Fruticultura 25: 210-212.

RICHARDSON EA et al. 1975. Pheno-crimatografy of spring peach bud development. HortScience 10: 236-237.

RODRÍGUEZ AR. 1995. Multiple regression models for the analysis of potential cultivation areas for Japanese plums. HortScience 30: 605-610.

SCHWARTZ MD. 2003. Phenology: An Integrative Environmental Science. 1.ed. Hardcover: Tasks for Vegetation Science. 132p.

SILVEIRA SV et al. 2012. Aspectos técnicos da produção de quivi. Bento Gonçalves: Embrapa. 84p. (Documentos 79).

SOUZA AP et al. 2011a. Comparison of methodologies for degree-day estimation using numerical methods. Acta Scientiarum. Agronomy 33: 391-400.

SOUZA AP et al. 2011b. Basal temperature and thermal sum in phonological phases of nectarine and peach cultivars. Pesquisa Agropecuária Brasileira 46: 1588-1596.

SPIEGEL-ROY P \& ALSTON FM. 1979. Chilling and post-dormant heat requirement as selection criteria for late flowering pears. Journal of Horticultural Science 54: 115-120.

VILLA NOVA NA et al. 1972. Estimativa de graus-dia acumulados acima de qualquer Tb em função das temperaturas máxima e mínima. Ciência da Terra 30: 1-8. 